\section{USO DE INTERNET EN PACIENTES DIABÉTICOS QUE BUSCAN INFORMACIÓN SOBRE SU ENFERMEDAD. LIMA, PERÚ, 2014}

\author{
INTERNET USE AMONG DIABETIC PATIENTS \\ SEARCHING FOR INFORMATION ABOUT \\ THEIR DISEASE. LIMA, PERÚ, 2014
}

Katherine Cántaro1,a , Percy Mayta-Tristán²,b

Sr. Editor. Hemos leído con interés el estudio publicado por Rivas-Nieto et al. realizado en un hospital del Ministerio de Salud, en 206 pacientes con diabetes, hipertensión y dislipidemia, donde reportan que el $13 \%$ de los pacientes habían buscado información de salud en Internet, además de discutir la potencial aplicación de las tecnologías de información y comunicación en el manejo de las enfermedades ${ }^{(1)}$.
Se realizó un estudio transversal donde se evaluó 464 pacientes con diabetes tipo 2, mayores de 18 años, con más de 6 meses de enfermedad, atendidos en el Centro de Atención Integral de Diabetes e Hipertensión (CEDHI) del Seguro Social (EsSalud) en Lima, Perú, durante el segundo semestre del 2014, previa aprobación de los comités de ética del Hospital Nacional Edgardo Rebagliati Martins y de la Universidad Peruana de Ciencias Aplicadas. Se midió el uso de diferentes fuentes para acceder a la información sobre diabetes, y entre ellas el uso de Internet.

El $20,3 \%$ de la muestra usó Internet para buscar información sobre diabetes, además, se encontró asociación con la edad (< 60 años), mayor nivel educativo, familiares o padres con diabetes, y estar en tratamiento dietético, con ejercicio o hipoglicemiantes orales, no se encontró asociación según sexo o tiempo de enfermedad (Tabla 1).

Tabla 1. Asociación entre el uso de Internet para buscar información sobre diabetes y las características de los pacientes con diabetes atendidos en el Centro de Hipertensión y Diabetes, EsSalud, Lima, 2014

\begin{tabular}{|c|c|c|c|c|c|c|c|}
\hline \multirow{2}{*}{ Características } & \multicolumn{2}{|c|}{ Total } & \multicolumn{2}{|c|}{ Usa Internet } & \multicolumn{2}{|c|}{ Modelo crudo* } & \multirow[b]{2}{*}{$p$} \\
\hline & $\mathbf{n}$ & $(\%)$ & $\mathbf{n}$ & $(\%)$ & $\mathbf{R P}$ & (IC95\%) & \\
\hline \multicolumn{8}{|l|}{ Sexo } \\
\hline Varón & 220 & $(47,5)$ & 52 & $(23,6)$ & 1,37 & $(0,95-1.97)$ & 0,092 \\
\hline Mujer & 243 & $(52,5)$ & 42 & $(17,3)$ & 1,00 & & \\
\hline \multicolumn{8}{|l|}{ Edad } \\
\hline$<60$ años & 165 & $(35,6)$ & 16 & $(10,9)$ & 2,84 & $(1,69-4,76)$ & $<0,001$ \\
\hline 61 a 70 años & 151 & $(32,6)$ & 27 & $(17,9)$ & 1,64 & $(0,92-2,92)$ & 0,091 \\
\hline > 70 años & 147 & $(31,8)$ & 51 & $(30,9)$ & 1,00 & & \\
\hline \multicolumn{8}{|l|}{ Pareja } \\
\hline Soltero/Viudo/Separado & 147 & $(31,8)$ & 21 & $(14,3)$ & 1,62 & $(1,04-2,52)$ & 0,034 \\
\hline Casado/conviviente & 316 & $(68,3)$ & 73 & $(23,1)$ & 1,00 & & \\
\hline \multicolumn{8}{|l|}{ Educación } \\
\hline Posgrado & 23 & $(4,97)$ & 12 & $(52,2)$ & 19,04 & $(4,59-79,04)$ & $<0,001$ \\
\hline Universitaria & 127 & $(27,4)$ & 44 & $(34,7)$ & 12,65 & $(3,15-50,72)$ & $<0,001$ \\
\hline Técnica & 98 & $(21,2)$ & 22 & $(22,5)$ & 8,19 & $(1,99-33,80)$ & 0,004 \\
\hline Secundaria & 142 & $(30,7)$ & 14 & $(9,9)$ & 3,60 & $(0,84-15,43)$ & 0,085 \\
\hline Sin educación/primaria & 73 & $(15,8)$ & 2 & $(2,7)$ & 1,00 & & \\
\hline \multicolumn{8}{|l|}{ Antecedentes de diabetes } \\
\hline Familiares con diabetes & 323 & $(69,8)$ & 58 & $(42,7)$ & 3,89 & $(2,70-5,60)$ & $<0,001$ \\
\hline Padres con diabetes & 181 & $(39,2)$ & 50 & $(27,6)$ & 1,81 & $(1,26-2,60)$ & 0,001 \\
\hline \multicolumn{8}{|l|}{ Tiempo de enfermedad } \\
\hline$>12$ años & 144 & $(31,9)$ & 29 & $(20,1)$ & 0,89 & $(0,58-1,37)$ & 0,597 \\
\hline 5-12años & 148 & $(32,8)$ & 28 & $(18,9)$ & 0,84 & $(0,54-1,30)$ & 0,424 \\
\hline 0-4años & 159 & $(35,3)$ & 33 & $(22,6)$ & 1,00 & & \\
\hline \multicolumn{8}{|l|}{ Tratamiento de diabetes } \\
\hline Dieta & 269 & $(58,2)$ & 65 & $(24,2)$ & 1,67 & $(1,11-2,49)$ & 0,013 \\
\hline Ejercicio & 158 & $(34,2)$ & 43 & $(27,2)$ & 1,65 & $(1,15-2,37)$ & 0,006 \\
\hline Hipoglicemiantes orales & 357 & $(77,3)$ & 80 & $(22,4)$ & 1,81 & $(1,05-3,12)$ & 0,033 \\
\hline Insulina & 71 & $(15,4)$ & 20 & $(28,2)$ & 1,51 & $(0,99-2,31)$ & 0,058 \\
\hline \multicolumn{8}{|l|}{ Hipertensión } \\
\hline Sí & 304 & $(65,8)$ & 57 & $(18,8)$ & 0,80 & $(0,56-1,16)$ & 0,235 \\
\hline No & 158 & $(34,2)$ & 37 & $(23,4)$ & 1,00 & & \\
\hline
\end{tabular}

* Modelo de regresión de Poisson con varianza robusta. RP: razón de prevalencia

\footnotetext{
1 Escuela de Nutrición, Universidad Peruana de Ciencias Aplicadas. Lima, Perú.

Escuela de Medicina, Universidad Peruana de Ciencias Aplicadas. Lima, Perú.

Nutricionista; ${ }^{b}$ médico cirujano

Recibido: 15-09-15 Aprobado: 21-10-15
}

Citar como: Cántaro K, Mayta-Tristán P. Uso de Internet en pacientes diabéticos que buscan información sobre su enfermedad. Lima, Perú, 2014 [carta]. Rev Peru Med Exp Salud Publica. 2016;33(1):173-4. doi: 10.17843/rpmesp.2016.331.1883 
El uso de Internet no solo es importante como una fuente de información, sino que puede ser usado en el día a día del paciente con diabetes, en la promoción y control de su enfermedad (2), se ha extendido en forma gradual, siendo cada vez mayor su uso en personas mayores ${ }^{(3)}$; sin embargo, su manejo está condicionado por el nivel educativo y el acceso a este servicio, lo que finalmente depende del nivel económico de las personas ${ }^{(4)}$.

En este contexto, a diferencia del estudio de RivasNieto et al. realizado en un hospital del Ministerio de Salud (1), se encontró que en EsSalud hay una mayor proporción de uso de Internet para buscar información, lo que probablemente tenga relación con el mayor nivel educativo de esta población. La implementación de estrategias que usen Internet para el manejo de pacientes con diabetes, tendrían mayor posibilidad de uso en hospitales de EsSalud que del Ministerio de Salud, y probablemente mayor en clínicas privadas.

Fuentes de financiamiento: autofinanciado.

Conflictos de interés: los autores declaran no tener conflictos de interés.

\section{REFERENCIAS BIBLIOGRÁFICAS}

1 Rivas-Nieto A, Málaga G, Ruiz-Grosso P, Huayanay-Espinoza C, Curioso WH. Uso y percepciones de las tecnologías de información y comunicación en pacientes con hipertensión arterial, dislipidemia o diabetes, de un hospital nacional de Lima, Perú. Rev Peru Med Exp Salud Publica. 2015;32(2):283-8.

2 Powers MA, March SB, Evert A. Use of internet technology to support nutrition and diabetes self-management care. Diabetes Spectr. 2008;21(2):91-9.

3 Kummervold PE, Chronaki CE, Lausen B, Prokosch HU, Rasmussen J, Santana S, et al. eHealth trends in Europe 2005-2007: a population-based survey. J Med Internet Res. 2008;10(4):e42. doi: 10.2196/jmir.1023.

4 Henriquez-Camacho C, Losa J, Miranda JJ, Cheyne NE. Addressing healthy aging populations in developing countries: unlocking the opportunity of eHealth and mHealth. Emerg Themes Epidemiol. 2014;11(1):136. doi: 10.1186/s12982014-0021-4.

\footnotetext{
Correspondencia: Katherine Cántaro

Dirección: Francisco de Zela 1039 Int. G Jesús María. Lima, Perú Teléfono: (511) 994869165

Correo electrónico:pcnukcan@upc.edu.pe
}

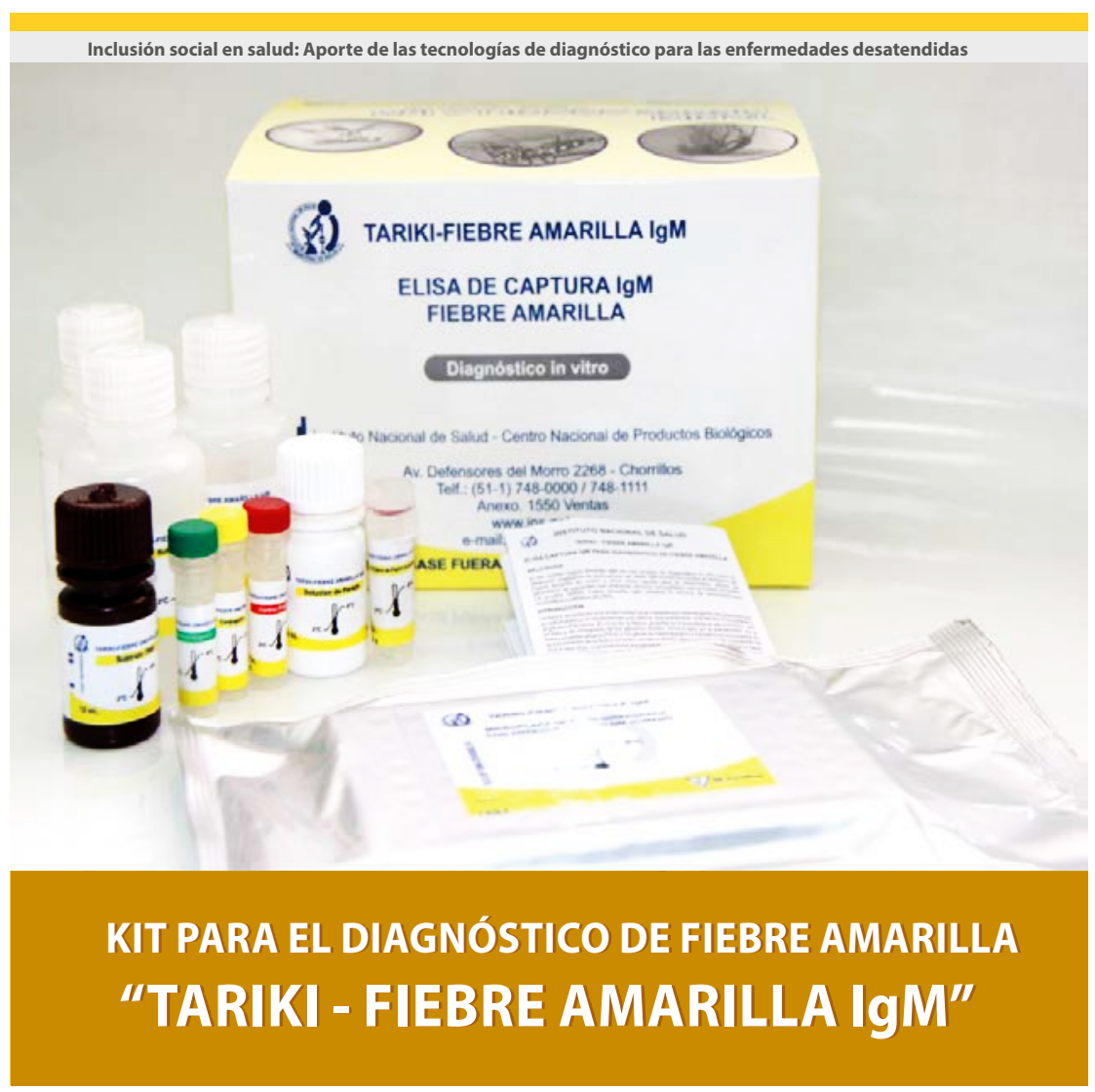

